\title{
Appearance of Ferromagnetic Property for Si Nanopolycrystalline Body and Vanishing of Electrical Resistances at Local High Frequencies
}

\author{
Taku Saiki $(\mathbb{D}$, Yukio Iida, and Mitsuru Inada \\ Faculty of Engineering Science, Kansai University, 3-3-35 Yamate, Suita, Osaka 564-8680, Japan \\ Correspondence should be addressed to Taku Saiki; tsaiki@kansai-u.ac.jp
}

Received 17 July 2018; Accepted 8 October 2018; Published 25 November 2018

Guest Editor: Vidyadhar Singh

Copyright @ 2018 Taku Saiki et al. This is an open access article distributed under the Creative Commons Attribution License, which permits unrestricted use, distribution, and reproduction in any medium, provided the original work is properly cited.

\begin{abstract}
Reduction in the skin effect for the sintered Si nanopolycrystalline body as an electricity conductor at a high frequency due to its nanostructure was studied. Singular disappearance of electrical resistances near a local high magnetic harmonic frequency of a few $\mathrm{MHz}$ was observed. This phenomenon has not been observed for conventional ferromagnetic metals. The measured electrical resistances changed to almost $0 \mathrm{~m} \Omega$ at room temperature. At the same time, negative resistance of the sintered $\mathrm{Si}$ nanopolycrystalline body was observed. It will be applicable to electronic transmittance lines or semiconductors. Numerical calculation was also performed on the electrical resistance with frequency dependency while considering the electric field and magnetic field in the sintered Si nanopolycrystalline body. The experimental and calculated results are compared. The calculation could explain the variation of the relative permittivity of the Si nanopolycrystalline body and the phenomenon for the theoretical disappearance of the resistivity at the $\mathrm{MHz}$ frequency. Reduced $\mathrm{Si}$ nanoparticles from $\mathrm{SiO}_{2}$ powder were synthesized by laser ablation in liquid. A Si nanopolycrystalline body made of the reduced Si nanoparticles was fabricated. It was found by measuring the magnetization property of the body of the sintered Si nanopolycrystalline body which is ferromagnetic. Dangling bonds (unpaired electrons) have long been known to occur due to defects in Si crystals. Perfect Si without defective crystals has no dangling bonds. However, Si nanoparticles have many dangling bonds. High-density dangling bonds cause the sintered Si nanopolycrystalline body to have ferromagnetism. In this study, the density of the unpaired electrons in the sintered Si nanopolycrystalline body was observed using ESR. It has been clarified that the Si nanopowder and the sintered Si nanopolycrystalline body have numerous dangling bonds. Both densities of the dangling bonds were evaluated.
\end{abstract}

\section{Introduction}

Reduction of AC resistance owing to the skin effect at high frequency from $\mathrm{MHz}$ to $\mathrm{GHz}$ in electronic devices or transmittance lines has been attracting attention now due to its ability to realize low-power consumption. We can expect that low-loss electric power lines and low-power-consumption electronic devices with low heat generation can be achieved by developing metals and semiconductors that have the reduced skin effect [1-4]. Research has also been conducted on the reductions in the skin effect for electric power lines using litz wire [2] or the negative permittivity of magnetic materials $[5,6]$. A multilayered material, which consists of different materials, such as metal and magnetic, is used for reducing the skin effect when a high-frequency current is conducted. This makes the effective permittivity equal zero when magnetic resonance occurs in magnetic material, negative permittivity at the real part of the relative permeability appears, and magnetic flux is canceled in multilayered materials [6].

At the same time, a technology has been developed to make electrical circuits at low temperatures of below $470 \mathrm{~K}$ using paste or ink consisting of $\mathrm{Au}[7], \mathrm{Ag}[8,9], \mathrm{Cu}[10]$, $\mathrm{Fe}[11,12], \mathrm{Al}[11,12]$, and $\mathrm{Mg}[12]$ nanoparticles owing to the phenomena of melting point depression. The metal nanoparticle paste and ink have often been sintered by furnace or pulsed light-like Xe flash lamp $[8,9]$ or laser $[12,13]$ in a short time. A free-form electrical circuit 
can be made in a short time and for low cost by using them. It is possible to make solids maintaining or improving the property of the nanoparticles through sintering.

Moreover, many methods to produce metal or nonmetal nanoparticles exist. Of all of them, the method for producing the metal nanoparticles using laser ablation in liquids with high speed and low cost has especially attracted attention [11-19]. Many kinds of magnetic nanoparticles [20-32] (such as $\mathrm{Fe}$ [20-24], iron oxide [26], Au [27], Ni [26], and Pd [27-29] nanoparticles) have already been researched. Ferromagnetism materials will be applicable to highfrequency core inductors $[31,32]$. However, to realize our goal, only some materials meet the following conditions for the energy transfer or the semiconductor: (1) low resistivity, (2) low cost, and (3) abundance. Thus, we choose Si, which consists of a single atom and is used in standard semiconductors and solar panels. Some applications of Si are semiconductors and solar panels. Additionally, Si nanoparticles, which can emit visible light owing to the quantum size effects, can be used in color displays [15-19, 33, 34]. Also, researches on the spin wave propagation in Si nanoparticles $[35,36]$, optical switch for optical computing using carrier effect by controlling the transparency of the material [37-40], and application to air cells [13] are being conducted right now.

Unpaired electrons, i.e., dangling bonds, due to impurities in Si substrates have already been studied by many researchers. $\mathrm{E}^{\prime}$ and $\mathrm{Pb}$ defects due to surface oxidation of $\mathrm{Si}$ have already been confirmed [41-51]. Also, a theoretical research reports that ferromagnetism of the Si material is generated by interaction between dangling bonds that become unpaired electrons [35]. However, the ferromagnetism of bulk Si has not been observed experimentally until now. High-density unpaired electrons are needed to generate ferromagnetism. Similar to Si material with crystal defects, the sintered $\mathrm{Al}$ polycrystalline bodies have been proven to be ferromagnetic even though common Al bulk is nonmagnetic [30]. Common nonmagnetic metals have been proposed to be able to change to being ferromagnetic when we make solids from nanoparticles.

The goal of our research is to develop low-powerconsuming semiconductor devices by applying the prepared Si nanopolycrystalline with a reduced skin effect to semiconductors. We have recently fabricated nanostructured metals by the bottom-up process [11-13]. A sintered metal nanopolycrystalline body with low resistivity is a new material, and the resistivity has not been adequately investigated.

In this study, we investigated if sintered Si nanopaste is a ferromagnetic material. We also report on a significant reduction in the resistance at a specific frequency that was also observed.

\section{Result}

2.1. Measurement by SEM. We observed the structure of the sintered Si nanopolycrystalline body experimentally. A SEM image of a sintered Si nanopolycrystalline body is shown in Figure 1. It has been found by EDX analysis that the $\mathrm{Si}$ secondary particles consist of $\mathrm{Si}$ atoms. The mixed paste contains $6 \%$ of $\mathrm{Ag}$ atoms for all the atoms when we make

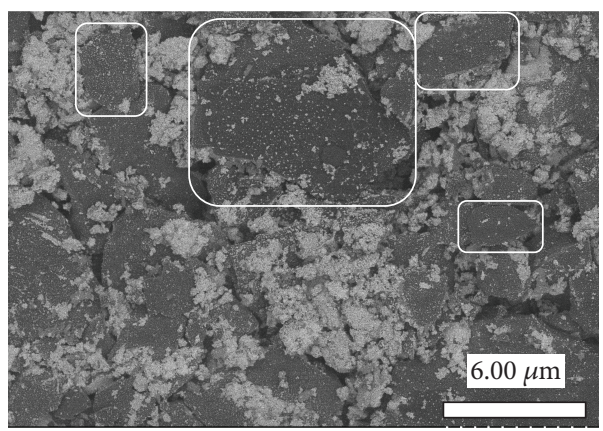

(a) Reflection electron image obtained by SEM (magnification: 5000 times)

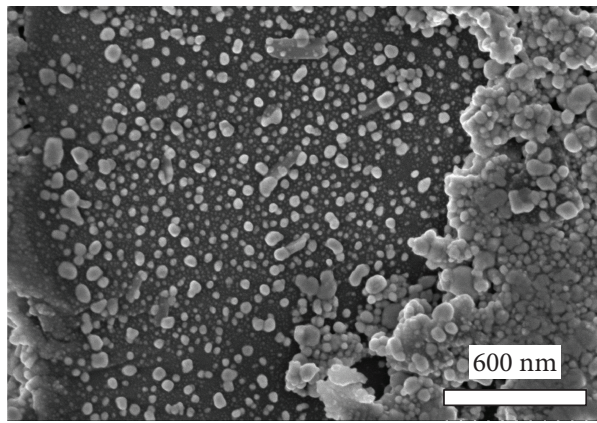

(b) Secondary electron image obtained by SEM (magnification: 50000 times)

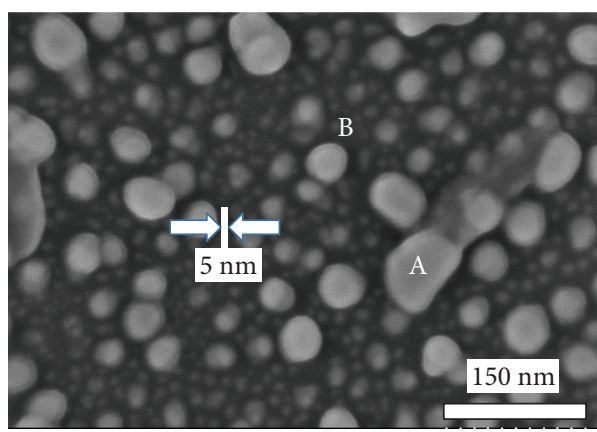

(c) Secondary electron image obtained by SEM (magnification: 200000 times)

FiguRe 1

Si nanopaste. After sintering, the amount will reduce to a few $\%$ because the Ag concentrated on the surface should be removed. It is hard to know the concentration. Numerous Si secondary particles below $10 \mu \mathrm{m}$ were observed as shown in white lines of Figure 1(a). The part surrounded by the white line is a secondary Si particle. The magnified SEM image in Figure 1(b) shows that this secondary particle is clearly composed of many minute Si nanoparticles. The expanded SEM image in Figure 1(c) shows that the Si nanoparticles are around a few $\mathrm{nm}$ although there is some variation in size. Figure 2 shows the results of EDX analysis by specific X-rays. Figures 2(a) and 2(b) show results for part $\mathrm{A}$ in black and part B in white. Si, O, and Ag atoms on the nanoparticles were measured at part $\mathrm{A}$ by specific $\mathrm{X}$-rays in Figure 2(a), and $\mathrm{Si}$ and $\mathrm{O}$ atoms on the nanoparticles were measured at part B by specific X-rays in Figure 2(b). Although oxygen appears strongly in the specific X-ray spectrum, it seems to be particularly attached to the surface of the Si nanoparticle and constitutes the interface of the secondary particle. Because the oxygen film is extremely thin, 


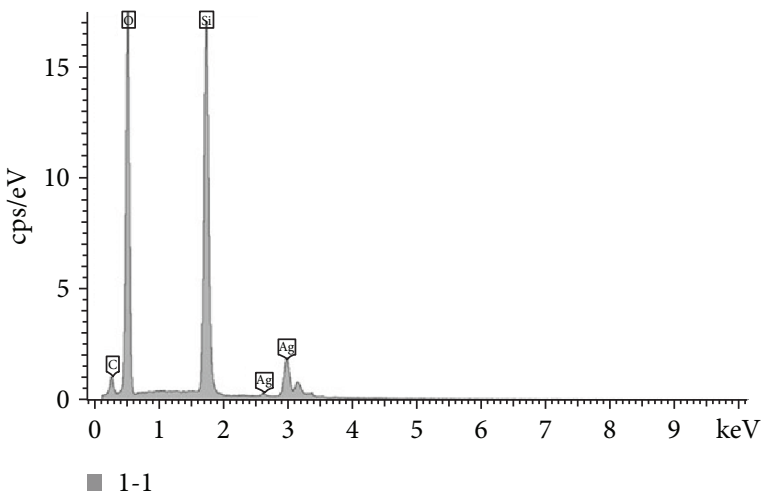

(a)

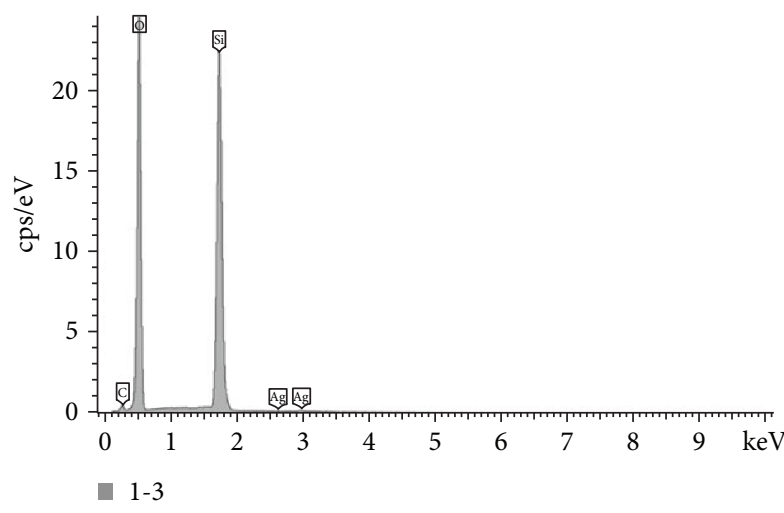

(b)

Figure 2: Results of EDX analysis by specific X-rays: (a) part A in Figure 1(c); (b) part B in Figure 1(c).

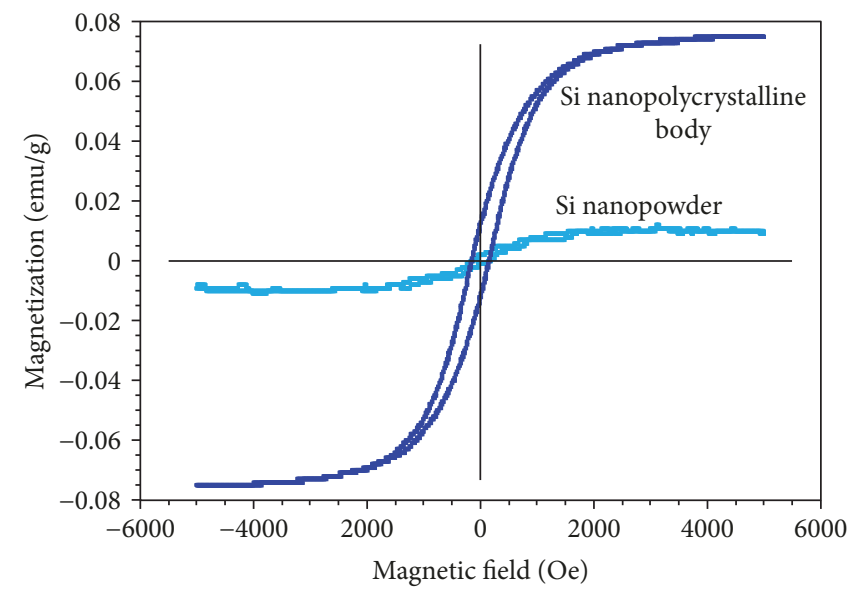

(a)

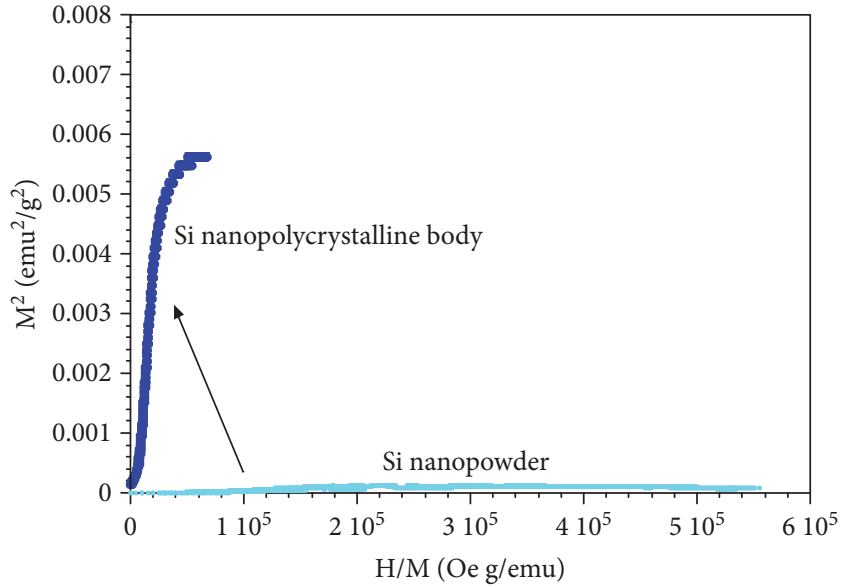

(b)

FIGURE 3: Magnetic property: (a) magnetization property; (b) Arrott plot.

the resistance does not increase. The diameter of the Si nanocrystals should not change during sintering. The oxygen film is thin and does not increase the resistance. It is clear from Figure 1(c) that secondary Si particles consist of numerous nanocrystals with a mean diameter of below $2 \mathrm{~nm}$ in three dimensions. The Si nanopolycrystalline body emits a photoluminescence with a peak wavelength of $550 \mathrm{~nm}$ excited by a violet light source due to the small size of the Si nanocrystals $[33,34]$.

2.2. Magnetization Property. Normal Si has an antimagnetic property. The magnetization property of the reduced $\mathrm{Si}$ nanoparticles and the sintered Si nanopolycrystalline body was measured by VSM at a room temperature $(293 \mathrm{~K})$ to determine if the sintered Si nanopolycrystalline body has ferromagnetism. The measured magnetization of reduced Si nanopowder and sintered $\mathrm{Si}$ is shown as Figure 3(a). Also, their Arrott plot is shown in Figure 3(b). Their magnetic parameters are shown in Table 1.

The measured maximum magnetization of the reduced $\mathrm{Si}$ nanoparticles was $0.011 \mathrm{emu} / \mathrm{g}$, and the measured maximum magnetization of the sintered Si nanopolycrystalline body
TABLE 1: Magnetic parameter.

\begin{tabular}{lcc}
\hline & $\begin{array}{c}\text { Saturated magnetization } \\
(\mathrm{emu} / \mathrm{g})\end{array}$ & $\begin{array}{c}\text { Corrective force } \\
(\mathrm{Oe})\end{array}$ \\
\hline Si nanopowder & 0.011 & 200 \\
Si nanocrystalline & 0.08 & 200 \\
Fe powder [21] & 210 (mean size: $150 \mathrm{~nm})$ & 280 \\
Fe oxide [25] & $74($ mean size: $30 \mathrm{~nm})$ & 700 \\
Ni-doped ZnO [26] & 4 & 90 \\
\hline
\end{tabular}

was $0.075 \mathrm{emu} / \mathrm{g}$. The magnetization of the sintered $\mathrm{Si}$ nanopolycrystalline body is 7 times larger than that of the reduced Si nanoparticles. The maximum magnetization of the conventional Fe bulk was $218 \mathrm{emu} / \mathrm{g}$. The evaluated coercive force of the sintered Si nanopolycrystalline body was 200 Oe. From the measured magnetization property, the sintered Si nanopolycrystalline body includes a ferromagnetic phase because of the residual magnetization and coercive force as shown in Figures 3(a) and 3(b). Because the magnetization curve is saturated at $1000 \mathrm{Oe}$ for the reduced 


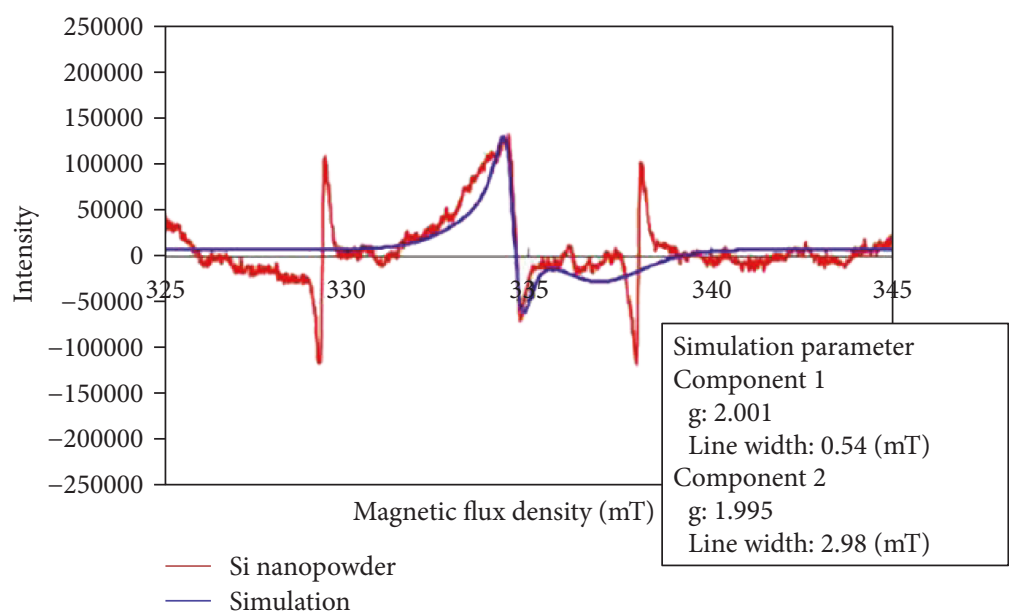

(a) Results of ESR analysis for Si nanopowder

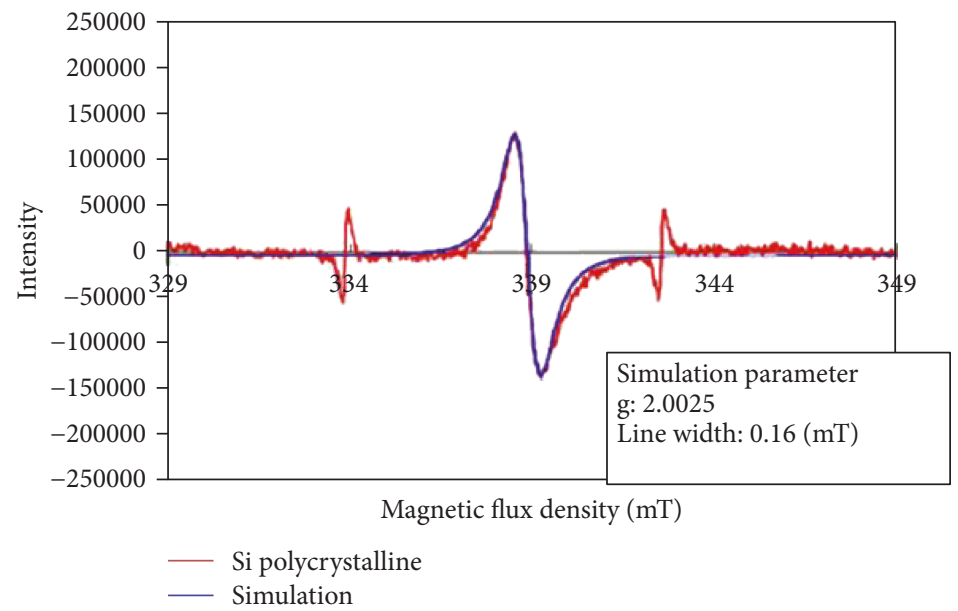

(b) Results of ESR analysis for sintered Si nanopolycrystalline

Figure 4

Si nanoparticles, there was no evident spontaneous magnetization, but there were many defects of $\mathrm{Si}$ atoms with magnetic moment in the material, which react with the outer magnetic field.

2.3. ESR Analysis. Densities of the dangling bond in the Si nanopowder and the Si nanopolycrystalline were measured by electron spin resonance (ESR) and then compared. Results of ESR analysis for the Si nanopowder and the sintered $\mathrm{Si}$ nanopolycrystalline are shown in Figures 4(a) and 4(b). The measured densities of the dangling bonds for the $\mathrm{Si}$ nanopowder and the $\mathrm{Si}$ nanopolycrystalline are shown in Table 2. The $g$ value for the Si nanopowder was 2.0000. It was considered from the $g$ value that the Si nanopowder contains the dangling bonds of the Si oxides. Judging from the observed peak signal, the Si nanopowder contains more than two components. However, the structure of the Si nanoparticle is not recognized. It should be a kind of Si oxide. The $g$ value for the Si nanopolycrystalline was 2.0025, which was between that for the Si crystals ( $g=2.0050 \sim 2.0060)$ and that for the Si oxides $(g=2.0000)$. It was found that intermediate dangling bonds between crystalline silicon and silicon oxide
TABLE 2: Result on evaluated density of dangling bond.

\begin{tabular}{lcc}
\hline & $g$ value & $\begin{array}{c}\text { Amount of dangling bond } \\
\left(\times 10^{13} \mathrm{spin} / \mathrm{g}\right)\end{array}$ \\
\hline Si nanopowder & 2.0000 & 8.3 \\
Si nanopolycrystalline & 2.0025 & 16.0 \\
\hline
\end{tabular}

exist in sintered Si nanopolycrystalline. $\mathrm{E}^{\prime}$ centers and $\mathrm{Pd}$ centers in the reference [41-45] could not be separated in the electron spin resonance (ESR) spectrum. The amount of the dangling bonds calculated from the integrated value of the ESR spectrum and the area strength of the standard material (known amount of radical: DPPH) is estimated to be $1 / 2$ that of the sintered Si nanopolycrystalline. Thus, it is concluded from this result that magnetization will be amplified up to seven times for one of the Si nanopowders because of the interaction between unpaired electrons called as exchange interactions in the sintered Si nanopolycrystalline.

2.4. Measurement of Resistivity. Measured resistance of the sintered $\mathrm{Si}$ as a function of frequency is shown in Figure 5. 


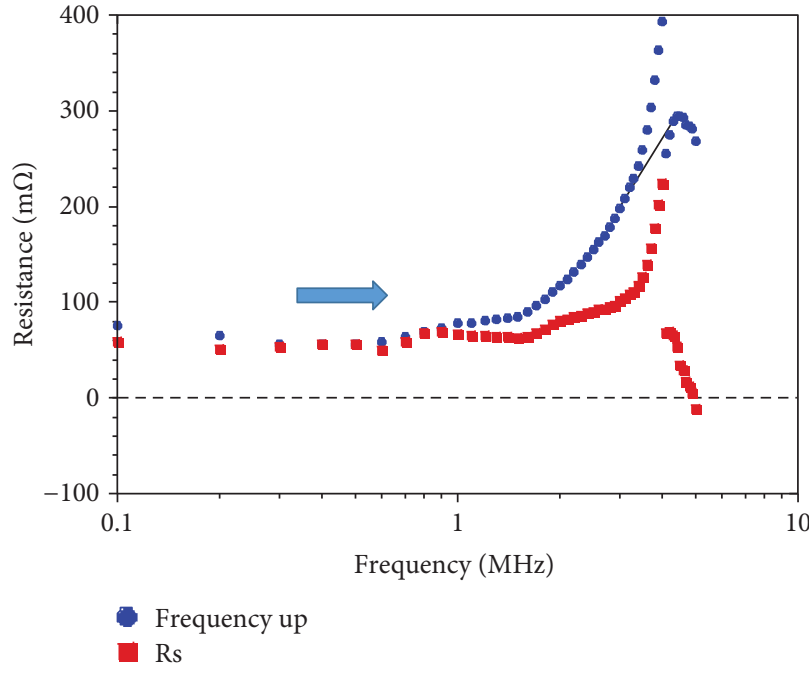

(a)

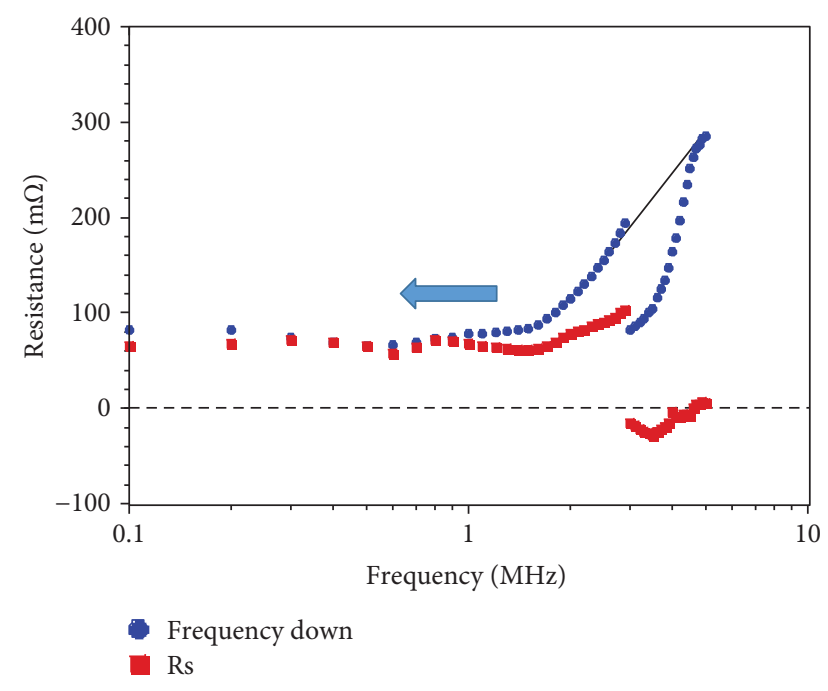

(b)

Figure 5: Measured resistivity as function of frequency when current at $5 \mathrm{MHz}$ was $10 \mathrm{~mA}$ : (a) increasing frequency; (b) decreasing frequency.

The measured resistance with increasing frequency of the sine wave signal is shown in Figure 5(a), and that with decreasing frequency of the sine wave signal is shown in Figure 5(b). The blue line shows the resistance measured with copper lines. The red line shows the resistance without the resistance of the copper lines. We observed a clear difference between the two results. Discontinuities of the resistances as a function of frequency were observed.

The resistance of the sintered Si nanopolycrystalline body increased with increasing frequency, and the resistance returned to $60 \mathrm{~m} \Omega$ at $4 \mathrm{MHz}$. After that, the resistance decreased continuously, as shown in Figure 5(a). The resistance of the sintered Si nanopolycrystalline body remained close to zero with decreasing frequency, and the resistance decreased around $-20 \mathrm{~m} \Omega$ at $3 \mathrm{MHz}$. After that, the resistance decreased continuously from $100 \mathrm{~m} \Omega$ as if the skin effect was weakened, as shown in Figure 5(b). The evaluated volume resistivity of the sintered $\mathrm{Si}$ nanopolycrystalline body was $1.2 \times 10^{-6} \Omega \mathrm{m}$ at $100 \mathrm{~Hz}$. A slight reduction in the resistance at $200 \mathrm{kHz}$ was also observed.

The measured resistance of the sintered Si depending on the current is shown in Figure 6. The resistances were measured with decreasing frequency of the sine wave signal. The measured self-inductances of the sintered $\mathrm{Si}$ nanopolycrystalline body are shown in the inset figures of Figures 6(a)-6(c). Thus, from the current data, magnetic resonance occurs at these frequencies, and the relative permeability changes rapidly around these frequencies. The singular radical vanishing of the resistivity in the Si nanopolycrystalline body at high magnetic-resonance frequencies should occur because the sintered Si pastes are ferromagnetic and exhibit negative permittivity around these frequencies. The self-inductance increased at $2.8 \mathrm{MHz}$, as shown in the inset of Figure 6(a). The self-inductance changed discontinuously at $200 \mathrm{kHz}, 2.8 \mathrm{MHz}$, and $5 \mathrm{MHz}$. The resistance of the sintered $\mathrm{Si}$ increased to $400 \mathrm{~m} \Omega$ at $2.8 \mathrm{MHz}$, as shown in
Figure 6(a). At the same time, the current decreased to half that at $100 \mathrm{kHz}$.

The measured resistances of the $\mathrm{Si}$ nanopolycrystalline body depending on the current are shown in Figure 6(d). The resistance at $3 \mathrm{MHz}$ was $190 \mathrm{~m} \Omega$ when the current was $0.17 \mathrm{~mA}$ and changed to near $-20 \mathrm{~m} \Omega$ when the current was changed from 2 to $8 \mathrm{~mA}$. Finally, the resistance was $0 \mathrm{~m} \Omega$ asymptotically when the current was changed from 10 to $18 \mathrm{~mA}$. The resistance at $5 \mathrm{MHz}$ was $-120 \mathrm{~m} \Omega$ when the current was $0.17 \mathrm{~mA}$ and was $0 \mathrm{~m} \Omega$ asymptotically when the current was changed from 3 to $8 \mathrm{~mA}$.

The resistance at high frequency was calculated numerically while considering the permittivity of the Si nanopolycrystalline and the electric field and magnetic field. From the results, the resistance clearly varies in accordance with the relative permittivity of 1 as a function of the frequency as shown by the dashed lines in Figures 6(a)-6(c). This analysis result seems to be extraordinary because the sintered $\mathrm{Si}$ is metal. Thus, the relative permeability should degrade to below 1 as in the case of normal metal bulk. The lower relative permeability means an antimagnetic property such as that appearing in conventional Al bulk. However, it was found from numerical calculation that the relative permeability of the sintered Si nanopolycrystalline body degraded to close to 1 .

Thus, the relative permittivity was changed so as to fit the measured resistance. The evaluated relative permittivities as function of frequency are shown in Figure 7. The resistance was recalculated using the relative permittivity as shown by the blue solid line in Figures $6(a)-6(c)$. The resistance increased to $400 \mathrm{~m} \Omega$ when the frequency was $2.5 \mathrm{MHz}$ as shown in Figure 6(a). This is because the generated magnetic field intensity is weak and the amplitude of the permittivity is maximized. It was thought that the phase of the permeability is inverted at $5 \mathrm{MHz}$, the real part of the relative permeability changed to be -1 , and the 


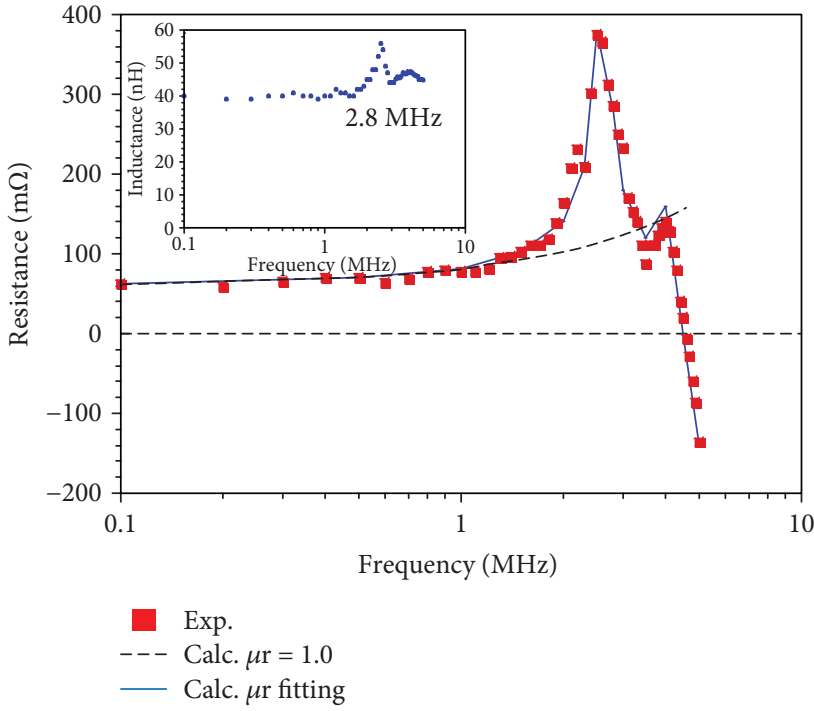

(a)

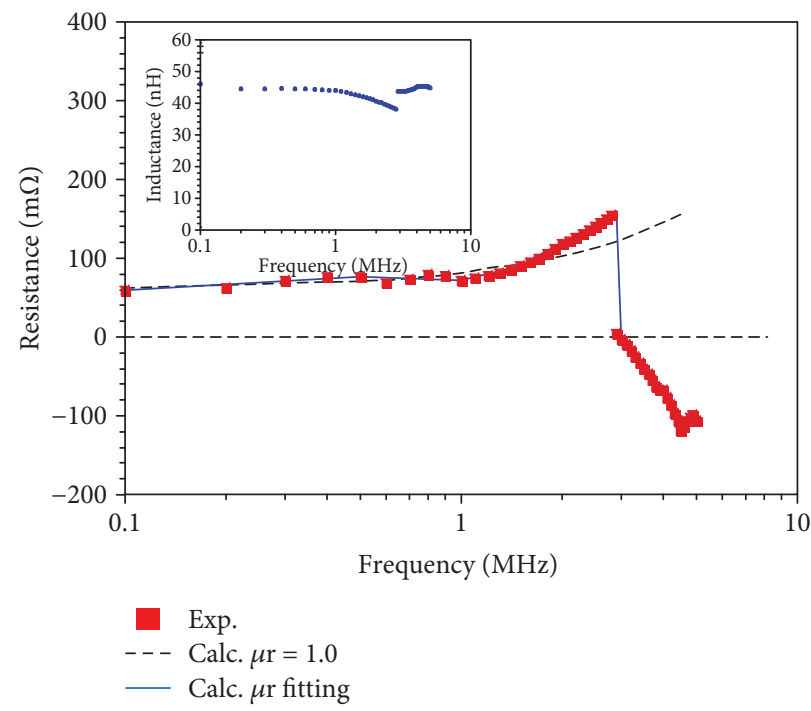

(c)

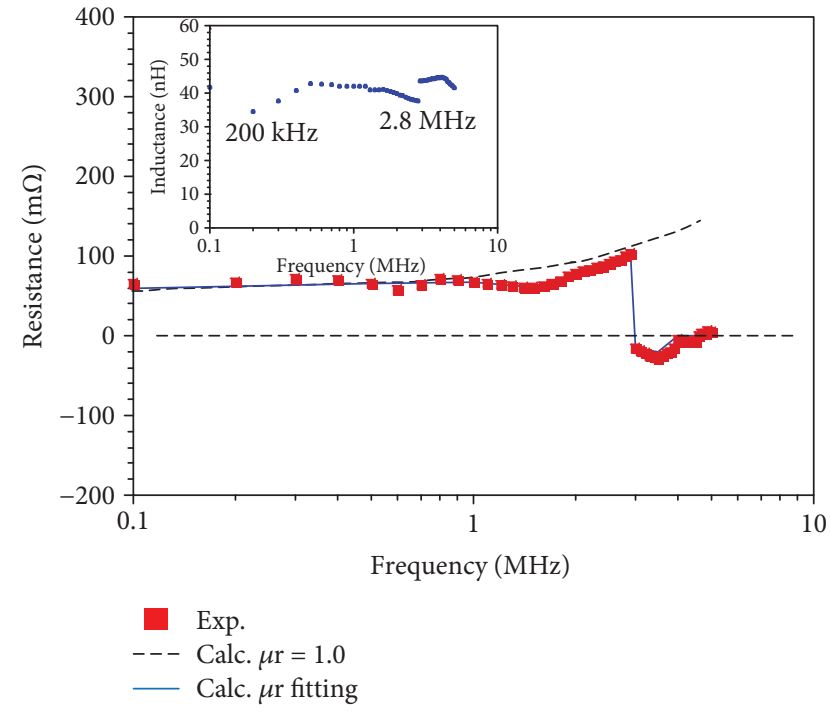

(b)

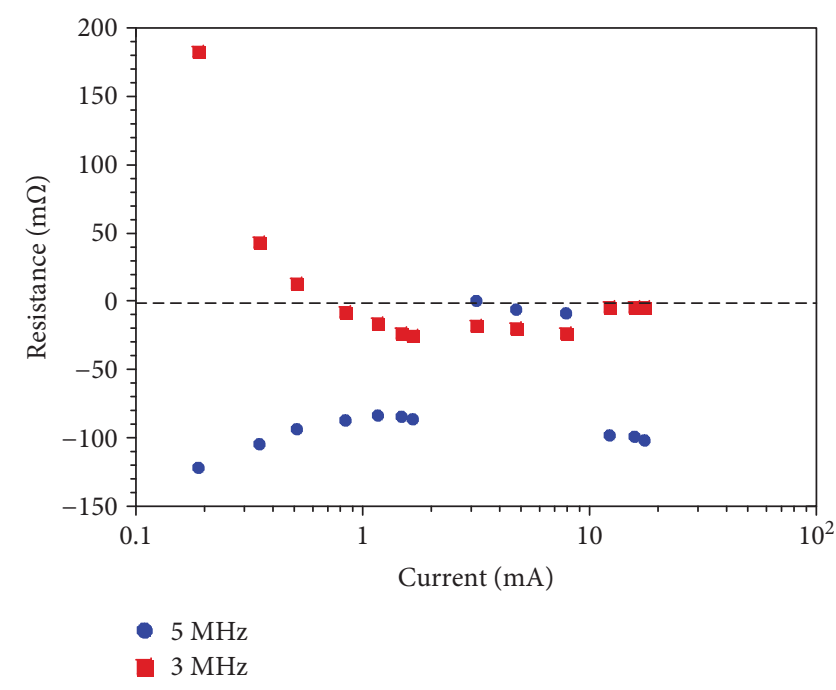

(d)

Figure 6: Results on measured resistance as function of frequency. Currents at $5 \mathrm{MHz}$ were (a) 0.17, (b) 10, and (c) $20 \mathrm{~mA}$. (d) Resistances with dependence on current.

direction of the electric field generated by the magnetic field is reversed.

For Figure 6(b), when the current increased, the intensity of the magnetic field increased by two orders of magnitude. The magnetic moment cannot keep up with the time change of the magnetic strength owing to the high frequency, and at $3 \mathrm{MHz}$, the real part of the relative permeability is a binary change of 1 or -1 . It was also found that the phase of the permeability was inverted at $1.5 \mathrm{MHz}$.

For Figure 6(c), when the current increased, the magnetic field intensity became stronger. The magnetic moment cannot keep up with the temporal change of magnetic intensity owing to the high frequency, but at $3 \mathrm{MHz}$, the real part of the relative permeability is a binary change of 1.5 on the low-frequency side and -1 on the highfrequency side.
In the case in Figure 6(a), the permeability was found to be consistent with that of the sintered $\mathrm{Al}$ having similar properties described in the reference [30], with the relative magnetic permeability at $2.5 \mathrm{MHz}$ evaluated to be 7 .

When the magnetic resonance occurred, the real part of the permeability changed to negative at $3 \mathrm{MHz}$, as show in Figure 8. The calculated results show that the real part of the permeability inside the sintered $\mathrm{Si}$ should be changed to $-\mu_{0}$. The term $E$ is the original electric field applied with the original sine wave signal, and $E_{B}$ is the electric field generated by the current-induced magnetic field. These directions are the same because of the phase inversion of $E_{B}$ when the real part of the relative permeability is negative. It was suggested that the electrons in the sintered $\mathrm{Si}$ nanopolycrystalline body were accelerated by the generated electric field. 


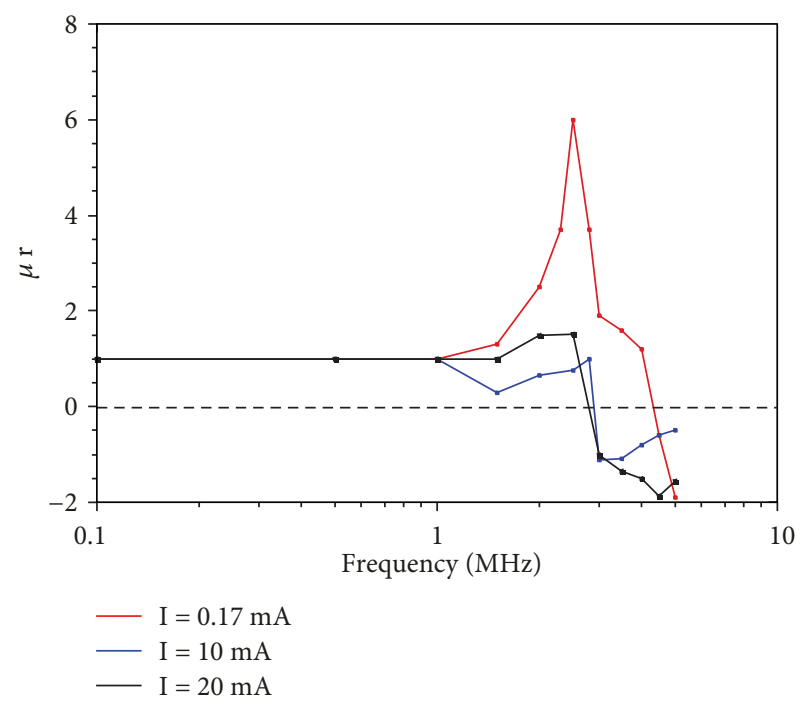

Figure 7: Evaluated relative permittivity as a function of frequency. Currents at $5 \mathrm{MHz}$ were $0.17,10$, and $20 \mathrm{~mA}$.

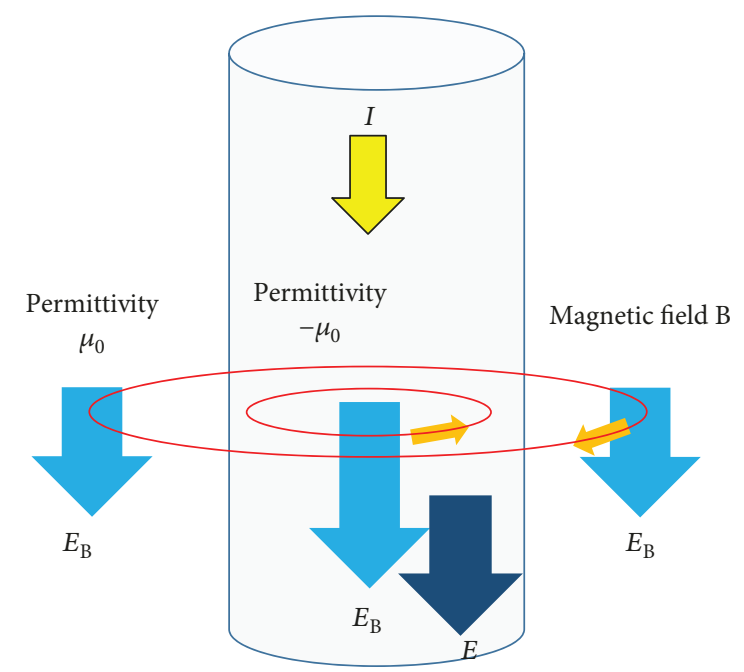

FIGURE 8: Spatial distribution of permeability when magnetic resonance occurred and real part of permeability changed to negative at $3 \mathrm{MHz}$.

However, at the same time, the sintered Si nanopolycrystalline body had a relative permeability of -1 . The numerically calculated result shows that the current does not flow linearly in micro scale of the sintered $\mathrm{Si}$ and generation of the eddy current is prevented due to the structure.

The resistance of a sintered $\mathrm{Ag}$ nanopolycrystalline body with nonferromagnetism did not become zero at the magnetic-resonance frequencies of a few MHz. Thus, this phenomenon is thought to be intrinsic for a metal nanopolycrystalline body with ferromagnetism.

Common metal has effective permeability below 1 , such as 0.5 , at the $\mathrm{MHz}$ level, and resistance becomes low due to the generated $E_{B}$. Thus, because the $E_{B}$ is weaker than that of metal in a ferromagnetic metal nanopolycrystalline body, the resistance does not degrade to zero.
It is considered that the resistances of almost all ferromagnetic metal nanopolycrystalline bodies change to zero at frequencies of a few $\mathrm{MHz}$ with no connection to the relative permeability.

However, resistance from 3 to $20 \mathrm{~mA}$ changed to close to zero by some cause. The resistance also changed to negative around $5 \mathrm{MHz}$ when the current was $20 \mathrm{~mA}$, as shown in Figure 6(d). The cause seems to be that the magnetic momentum of unpaired electrons at the surface of Si nanocrystals cannot follow the varying magnetic field at a high frequency of $5 \mathrm{MHz}$.

In these experiments, we did not observe the reduction of the skin effect by which the current distribution in the $\mathrm{Si}$ is close to uniform due to the nanostructure. We believe that sintered Si nanopaste with a Si nanopolycrystalline body will be applicable to magnetic materials.

2.5. Calculated Magnetic Field Intensity and Electric Field Intensity. Figure 9 shows the calculated results for the distribution of the magnetic field intensity at the profile of the $\mathrm{Si}$ nanopolycrystalline. The current is shown as the effective value. The thin direction of the material is the thickness direction, and the long $4 \mathrm{~mm}$ direction is the length direction. The magnetic field intensity at the center of both directions was calculated. It was found that a strong magnetic field is mainly generated near the surface in the direction perpendicular to the thickness direction. Also, a weak magnetic field of $1 / 10$ was generated in the direction perpendicular to the length direction.

It was found that when the effective value of the current flowing in the $\mathrm{Si}$ nanopolycrystalline was $0.17,10$, and $20 \mathrm{~mA}$, the calculated magnetic field intensity was 1,80 , and $160 \mathrm{~A} / \mathrm{m}$, respectively, in the direction perpendicular to the thickness direction.

Figure 10 shows the calculated distribution of the electric field, in which the direction of the electric field is the same as the direction of the current and the intensity is proportional to current. Here, the electrical field intensity in the longitudinal direction was normalized by the AC electrical field intensity added by a power source. When the normalized electric field is negative, it means that the current barely flows in the forward direction. When the normalized electric field is positive and larger than 1, this means that the flow of current is assisted by the electric field and the resistance is reduced.

For Figure 10(a), when the current is $0.17 \mathrm{~mA}$, the electric field intensity at the center continues to decrease from 0.1 to $4 \mathrm{MHz}$ and is lowest at $2.5 \mathrm{MHz}$. The real part of the permeability is changed to be negative by magnetic resonance. The distribution of the electric field intensity was dented downward, and the distribution reversed from 2.5 to $5 \mathrm{MHz}$. For Figure $10(\mathrm{~b})$, when the current increased to $10 \mathrm{~mA}$, the electric field intensity at the center continues to decrease from 0.1 to $2.9 \mathrm{MHz}$. The real part of the permeability is changed to be negative by magnetic resonance. The distribution of the electric field intensity was dented downward, and the distribution reversed from 3 to $5 \mathrm{MHz}$.

For Figure 10(c), when the current increased to $20 \mathrm{~mA}$, the electric field intensity at the center continues to decrease 


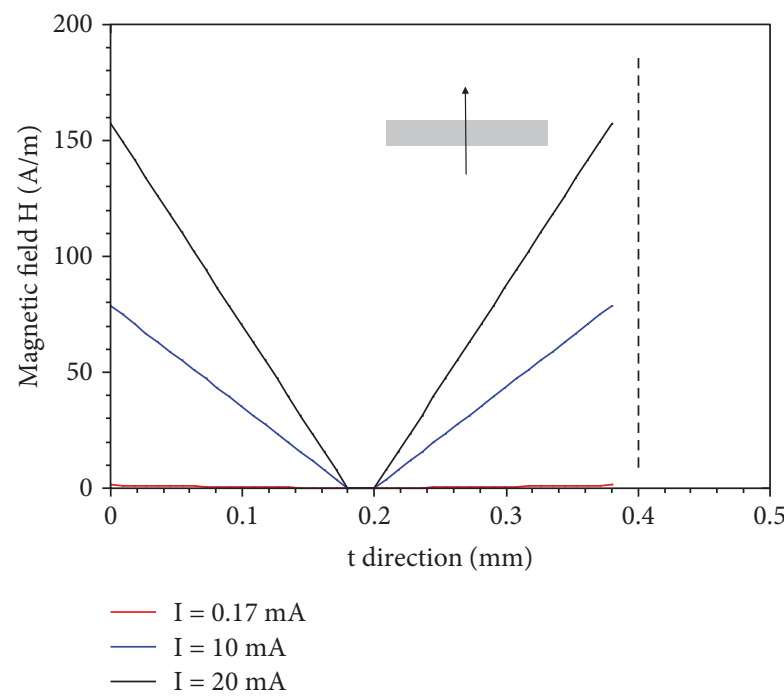

(a)

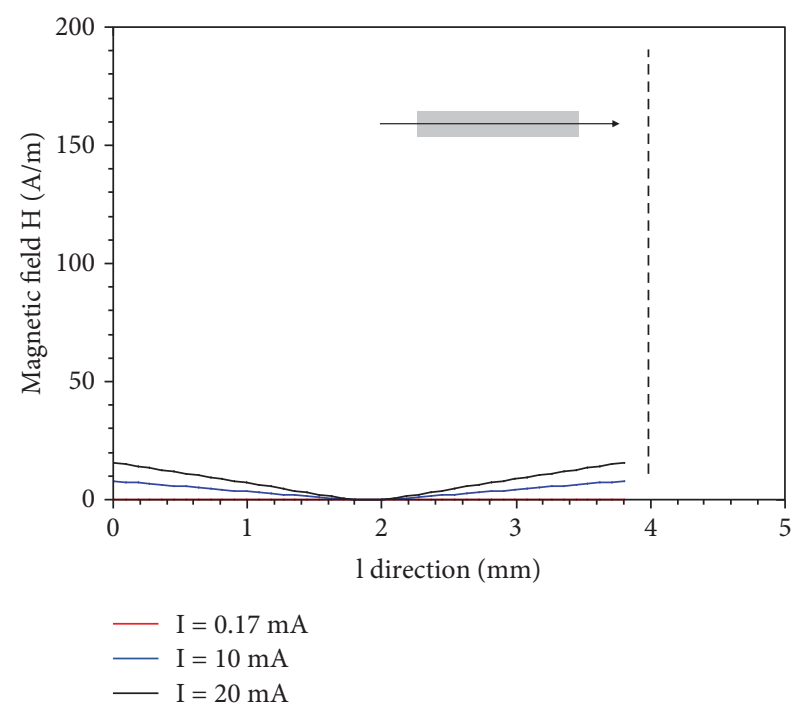

(b)

Figure 9: Calculated magnetic field intensity: (a) thickness direction; (b) length direction.

from 0.1 to $2.9 \mathrm{MHz}$ and is lowest at $2.9 \mathrm{MHz}$. The phase of the permeability is inverted to be negative by magnetic resonance. The distribution of the electric field intensity was dented downward, and the distribution reversed from 3 to $5 \mathrm{MHz}$.

\section{Conclusion}

Reduction in the skin effect for the sintered Si nanopolycrystalline for semiconductor material at a high frequency due to its nanostructure has been studied. Singular disappearance of electrical resistances near a local high magnetic harmonic frequency of a few $\mathrm{MHz}$ has been observed in the experiments. Negative resistance of the sintered Si nanopolycrystalline has also been observed. Numerical calculation has also been performed on the electrical resistance with frequency dependency while considering the electric field and magnetic field in the sintered Si nanopolycrystalline. The experimental and calculated results are compared. The calculation could theoretically explain the phenomenon of vanishing resistivity at the $\mathrm{MHz}$ frequency. It was found by measuring the magnetization property that the sintered Si nanopolycrystalline has ferromagnetism. The density of the unpaired electrons in the sintered Si nanopolycrystalline was observed using ESR. It has been recognized that the sintered Si nanopolycrystalline has numerous dangling bonds.

\section{Experimental Methods}

4.1. Production of Materials. The laser ablation method in liquid is described in the paragraph below. When laser pulses are irradiated onto metal oxides in liquid, the metal oxides melt and resolve, and the melted oxides are set outside the metal nanoparticles [11]. The surrounding liquid cools the metal nanoparticles rapidly. The experimental setup for laser pulse ablation is shown here [11]. A microchip Nd:YAG laser was used in this experiment. The maximum output averaged laser power was $250 \mathrm{~mW}$, the laser wavelength was $1064 \mathrm{~nm}$, the repetitive rate of the laser pulses was $18 \mathrm{kHz}$, and the pulse duration was $8 \mathrm{~ns}$. A beam with a diameter of $6 \mathrm{~mm}$ $\left(1 / e^{2}\right)$ was focused using a lens with a focal length of $50 \mathrm{~mm}$. Thus, the diameter of the focused beam was $20 \mu \mathrm{m}$ at the front of each glass bottle. Glass bottles with a size of $38 \mathrm{mml} \times 20 \mathrm{~mm}$ were used in the experiment. Reduced Si nanoparticles were produced by laser ablation in liquid. The liquid we used was pure water. The $\mathrm{SiO}_{2}$ powder (mean diameter of $5 \mu \mathrm{m}$, purity $99.9 \%$, Koujyund Chemical, Japan) was used to produce reduced $\mathrm{Si}$ nanoparticles. The $\mathrm{SiO}_{2}$ powder was mixed with the water in each glass bottle for the experiment. The glass bottle was set after the focused laser beam. The weight of the $\mathrm{SiO}_{2}$ powder was measured using an electronic force balance. The measured weight was $1.0 \mathrm{~g} .12 \mathrm{~mL}$ of pure water was placed in each glass bottle. Laser pulses were irradiated to the water with the metal oxide in the glass bottle for 10 minutes. Here, we neglect the oxidation at the surface of metal nanoparticles. A magnetic stirrer was used to mix the liquid. The color after laser irradiation changed to gray, which is close to the color of the reduced Si powder. The powder after irradiating laser pulses in the water was dried so as not to change chemically.

A sintered $\mathrm{Si}$ nanopaste ( $\mathrm{Si}$ nanopolycrystalline body) was made using the reduced Si nanoparticles. The dried Si nanopowders were mixed with $5 \mathrm{mg}$ of $\mathrm{Ag}$ nanopastes (NAG-10 Daiken Chemical); the viscosity of the paste was high. The size of the sintered Si nanopolycrystalline body was determined to be $4 \times 10 \times 0.3 \mathrm{~mm}$. The current was conducted in the longitudinal dimension, and the resistivity at a high frequency was measured. The Si paste was sintered using an electrical hot plate (CHP-170AN, ASONE) at $473 \mathrm{~K}$ ( $1 \mathrm{~min}$ ) and $533 \mathrm{~K}$ (4 min), enabling us to obtain sintered Si pastes.

4.2. Analysis of Material and Measurement Method. The magnetization properties of the reduced Si nanoparticles 

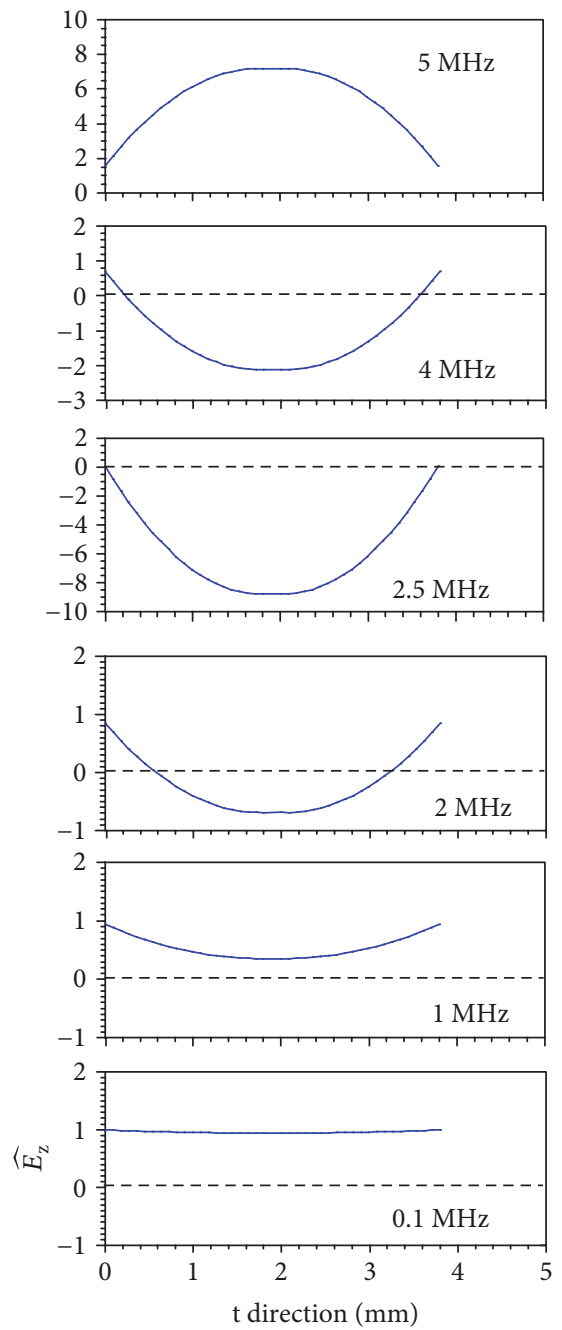

(a)
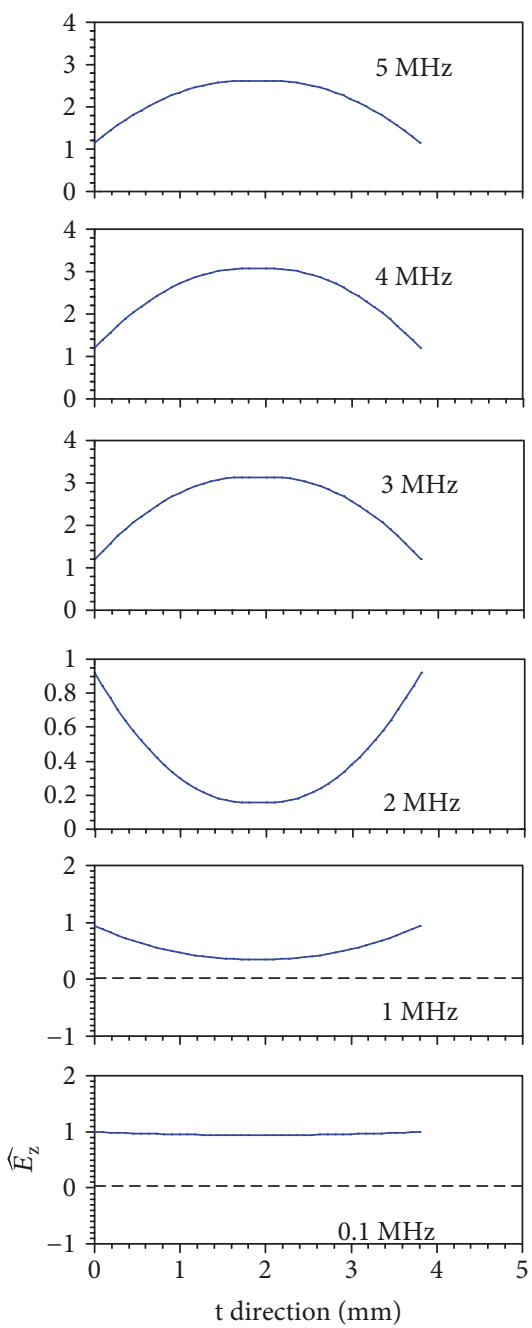

(b)
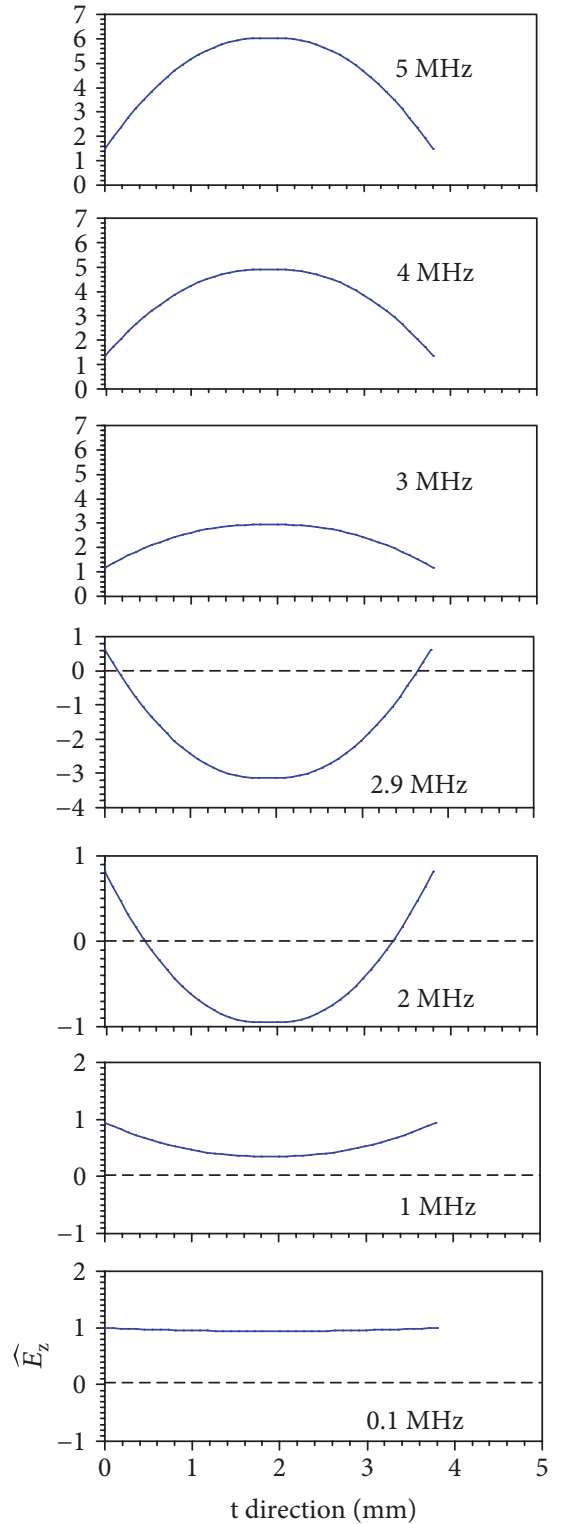

(c)

FIGURE 10: Calculated electric field: (a) $0.17 \mathrm{~mA}$, normalized by $E_{\mathrm{AC}}=0.0022 \mathrm{~V} / \mathrm{m}$; (b) $10 \mathrm{~mA}$, normalized by $E_{\mathrm{AC}}=0.013 \mathrm{~V} / \mathrm{m}$; (c) $20 \mathrm{~mA}$, normalized by $E_{\mathrm{AC}}=0.026 \mathrm{~V} / \mathrm{m}$.

and the sintered Si nanopolycrystalline body were measured using a vibrating sample magnetometer (VSM) (BHV-30T, Riken Denshi, Japan) at room temperature $\left(20^{\circ} \mathrm{C}\right)$. The sintered $\mathrm{Si}$ was observed by a scanning electron microscope (SEM) (S-4700 with low resolution and SU8240 with high resolution, Hitachi High-Technologies, Japan), and the existence of $\mathrm{Ag}, \mathrm{Si}$, and $\mathrm{O}$ atoms was analyzed by energy-dispersive X-ray spectrometry (EDX) (EMAX7000, Horiba, Japan).

We show the measurement condition for ESR analysis in Table 3.

FT-IR, S-FA200 type, JEOL was used for ESR analysis. The weight of the used sintered Si nanopolycrystalline body is $170 \mathrm{mg}$. The size was $20 \mathrm{~mm} \times 2.5 \mathrm{~mm} \times 1 \mathrm{~mm}$.
The resistance and inductance of the sintered Si pastes were measured using an LCR meter (3532-50 LCR, high tester, Hioki, Japan). The inductance and resistance from $42 \mathrm{~Hz}$ to $5 \mathrm{MHz}$ were measured. The inductors and electric power transmitters were assumed to have components of inductance and resistance. The phase angles of cascaded resistances and inductors in the stick-type sintered Si pastes were also measured.

4.3. Calculation for Skin Effect. We resolved the integration Maxwell-Faraday equation numerically and calculated the electric field generated by the current-generated magnetic field inside the metal. We observed spatial distribution of the electric field in the thickness and longitudinal directions. 
TABLE 3: Measurement condition.

\begin{tabular}{lc}
\hline Microwave frequency & $9.3-9.4 \mathrm{GHz}$ \\
Intensity of microwave & $12 \mathrm{~mW}$ \\
Range of maneuvering magnetic field & $20 \mathrm{mT}$ \\
Modulated magnetic field frequency & $100 \mathrm{kHz}$ \\
Modulated magnetic field amplitude & $1.0 \mathrm{mT}$ \\
Sampling time & $81.92 \mathrm{~ms}$ \\
Accumulation count & 3 times \\
Measurement temperature & $10 \mathrm{~K}$ (liquid He cooling) \\
Standard substance & 1-Diphenyl-2-picrylhydrazyl \\
Index & (DPPH) \\
\hline
\end{tabular}

The electric field was added to the original electric field applied from the sine wave signal source. The resistance was calculated with the recalculated electric field. We considered only the real part of the relative permeability. The special mesh was set to be 40 in both length and thickness directions. It was assumed that the permeability is uniform in the profile.

The current-generated magnetic field intensities in the directions of the length and thickness are given as

$$
\begin{aligned}
& H_{l}(r)=\frac{I r}{2 L t}, \\
& H_{t}(r)=\frac{I r L}{2 t^{3}},
\end{aligned}
$$

where $I$ is the AC current and the $l$ and $t$ are the length and the thickness, respectively, in the profile of the Si nanopolycrystalline body. The effective resistance at a high frequency is given as

$$
R_{\mathrm{hef}}=R_{\mathrm{DC}}\left(1+\frac{E_{\mathrm{Bav}}}{E_{\mathrm{AC}}}\right)
$$

where $R_{\mathrm{DC}}$ is the $\mathrm{DC}$ resistance, the $E_{0}$ is the original $\mathrm{AC}$ electric field intensity, and $E_{\mathrm{Bav}}$ is the averaged electrical field intensity generated by the current-generated magnetic field intensity. The resistance was calculated using the ratio of the electric field generated by the current-generated magnetic field to the original AC electric field. $R_{\mathrm{DC}}$ was set to be $60 \mathrm{~m} \Omega$.

\section{Data Availability}

No data were used to support this study.

\section{Conflicts of Interest}

The authors declare that they have no competing interests.

\section{Authors' Contributions}

T. S., Y. I., and M. I. contributed to the experimental design, data analyses and interpretation of the findings, and preparation of the manuscript. All authors approved the final version of the manuscript.

\section{References}

[1] D. G. Fink and H. W. Beatty, Standard Handbook for Electrical Engineers, McGraw Hill, 11 edition, 1978.

[2] C. R. Sullivan, "Optimal choice for number of strands in a litz-wire transformer winding," IEEE Transactions on Power Electronics, vol. 14, no. 2, pp. 283-291, 1999.

[3] H. Lamb, "On electrical motions in a spherical conductor," Philosophical Transactions of the Royal Society of London, vol. 174, pp. 519-549, 1883.

[4] W. H. Hayt, Engineering Electromagnetics, McGraw-Hill, 5th edition, 1989.

[5] Function Investigation Committee of Nanoscale Magnetic Material, Nanostructured Magnetic Materials -Physics, Function, Design-, Kyoritsu Publishing, Tokyo, Japan, 2010, Chapter 1 and 2.

[6] K. Yamada, K. Kim, and M. Yamaguchi, "Slit design consideration on the ferromagnetic RF integrated inductor," IEEE Transactions on Magnetics, vol. 42, no. 10, pp. 33413343, 2006.

[7] P. Buffat and J. P. Borel, "Size effect on the melting temperature of gold particles," Physical Review A, vol. 13, no. 6, pp. 2287-2298, 1976.

[8] W. H. Chung, H. J. Hwang, S. H. Lee, and H. S. Kim, “In situ monitoring of a flashlight sintering process using silver nano-ink for producing flexible electronics," Nanotechnology, vol. 24, no. 3, article 035202, 2013.

[9] W. M. Neill, C. Choi, C. Chang, and R. Malhotra, "On the selfdamping nature of densification in photonic sintering of nanoparticles," Scientific Reports, vol. 5, no. 1, article 14845, 2015.

[10] S.-H. Park, W.-H. Chung, and H.-S. Kim, "Temperature changes of copper nanoparticle ink during flash light sintering," Journal of Materials Processing Technology, vol. 214, no. 11, pp. 2730-2738, 2014.

[11] T. Saiki, T. Okada, K. Nakamura, T. Karita, Y. Nishikawa, and Y. Iida, "Air cells using negative metal electrodes fabricated by sintering pastes with base metal nanoparticles for efficient utilization of solar energy," International Journal of Energy Science, vol. 2, no. 6, pp. 228-234, 2012.

[12] T. Saiki, Y. Iida, K. Ri, M. Yoshida, and Y. Koga, "Electrical property of laser-sintered nanopastes with reduced metal nanoparticles prepared by laser ablation in liquids," Advances in Materials, vol. 3, no. 6, pp. 75-88, 2014.

[13] T. Saiki, K. Nakamura, J. Tokumoto, K. Nakamura, and T. Uematsu, "Fabrication of metal air cells using sintered Si nanopaste with reduced Si nanoparticles," The Review of Laser Engineering, vol. 45, no. 6, pp. 364-370, 2017.

[14] A. Henglein, "Physicochemical properties of small metal particles in solution: "microelectrode" reactions, chemisorption, composite metal particles, and the atom-to-metal transition," The Journal of Physical Chemistry, vol. 97, no. 21, pp. 5457-5471, 1993.

[15] L. T. Canham, "Silicon quantum wire array fabrication by electrochemical and chemical dissolution of wafers," Applied Physics Letters, vol. 57, no. 10, pp. 1046-1048, 1990.

[16] V. Švrček, T. Sasaki, Y. Shimizu, and N. Koshizaki, "Blue luminescent silicon nanocrystals prepared by ns pulsed laser ablation in water," Applied Physics Letters, vol. 89, no. 21, article 213113, 2006.

[17] K. Saitow and T. Yamamura, "Effective cooling generates efficient emission: blue, green, and red light-emitting Si 
nanocrystals," Journal of Physical Chemistry C, vol. 113, no. 19, pp. 8465-8470, 2009.

[18] I. Umezu, A. Sugimura, M. Inada, T. Makino, K. Matsumoto, and M. Takata, "Formation of nanoscale fine-structured silicon by pulsed laser ablation in hydrogen background gas," Physical Review B, vol. 76, no. 4, article 045328, 2007.

[19] L. Brus, "Electrinic wave functions in semiconductor clusters: experiment and theory," The Journal of Physical Chemistry, vol. 90, no. 12, pp. 2555-2560, 1986.

[20] T. Ogawa, Y. Ogata, R. Gallage et al., "Challenge to the synthesis of $\alpha^{\prime \prime}-\mathrm{Fe}_{16} \mathrm{~N}_{2}$ compound nanoparticle with high saturation magnetization for rare earth free new permanent magnetic material," Applied Physics Express, vol. 6, no. 7, article 073007, 2013.

[21] R. J. Joseyphusa, K. Shinodac, D. Kodamab, and B. Jeyadevanb, "Size controlled Fe nanoparticles through polyol process and their magnetic properties," Materials Chemistry and Physics, vol. 123, no. 2-3, pp. 487-493, 2010.

[22] M. Kamata, H. Kura, M. Takahashi, T. Ogawa, and T. Tanaka, "Direct synthesis of single crystalline-Fe nanoparticles with high saturation magnetization by mixed surfactant," IEEE Transactions on Magnetics, vol. 48, no. 11, pp. 39443946, 2012.

[23] H. Kura, M. Takahashi, and T. Ogawa, "Synthesis of monodisperse iron nanoparticles with a high saturation magnetization using an $\mathrm{Fe}(\mathrm{CO}) \mathrm{x}$-oleylamine reacted precursor," Journal of Physical Chemistry C, vol. 114, no. 13, pp. 5835-5838, 2010.

[24] S. Peng, C. Wang, J. Xie, and S. Sun, "Synthesis and stabilization of monodisperse Fe nanoparticles," Journal of the American Chemical Society, vol. 128, no. 33, pp. 1067610677, 2006.

[25] D. Cao, H. Li, L. Pan et al., "High saturation magnetization of $\gamma-\mathrm{Fe}_{2} \mathrm{O}_{3}$ nano-particles by a facile one-step synthesis approach," Scientific Reports, vol. 6, no. 1, pp. 1-9, 2016.

[26] X. He, W. Zhong, C.-T. Au, and Y. Du, "Size dependence of the magnetic properties of $\mathrm{Ni}$ nanoparticles prepared by thermal decomposition method," Nanoscale Research Letters, vol. 8, no. 1, p. 446, 2013.

[27] H. Hori, T. Teranishi, Y. Nakae, Y. Seino, M. Miyake, and S. Yamada, "Anomalous magnetic polarization effect of Pd and Au nano-particles," Physics Letters, vol. 263, no. 4-6, pp. 406-410, 1999.

[28] F. Aguilera-Granja, J. M. Montejano-Carrizales, and A. Vega, "Twining effects in the magnetism of small Pd clusters," Solid State Communications, vol. 133, no. 9, pp. 573-578, 2005.

[29] Y. T. Jeon and G. H. Lee, "Magnetism of the fcc Rh and Pd nanoparticles," Journal of Applied Physics, vol. 103, no. 9, article 094313, 2008.

[30] S. Masuda, T. Saiki, Y. Iida, and M. Inada, "High frequency core inductor using sintered aluminum nano-paste with aluminum nano-polycrystalline structure," in Conference on the Lasers and Electro-Optics (CLEO), San Jose, CA, USA, May 2017, paper STh1J.3.

[31] V. Korenivski, "GHz magnetic film inductors," Journal of Magnetism and Magnetic Materials, vol. 215-216, pp. 800806, 2000.

[32] P. Marín, D. Cortina, and A. Hernando, "Electromagnetic wave absorbing material based on magnetic microwires," IEEE Transactions on Magnetics, vol. 44, no. 11, pp. 39343937, 2008.
[33] N. Hill and K. Whaley, "Size dependence of excitations in silicon nanocrystals," Physical Review Letters, vol. 75, no. 6, pp. 1130-1133, 1995.

[34] T. Saiki and Y. Iida, "Fabrication of sintered Si nanopolycrystalline with reduced Si nanoparticles and property of photoluminescence in visible regime for sintered Si nanopolycrystalline by violet light excitation," American Journal of Nano Research and Applications, vol. 3, no. 5, pp. 82$88,2015$.

[35] S. Okada, K. Shiraishi, and A. Oshiyama, "Magnetic ordering of dangling bond networks on hydrogen-deposited $\mathrm{Si}(111)$ surfaces," Physical Review Letters, vol. 90, no. 2, 2003.

[36] P. N. Hai, S. Ohya, and M. Tanaka, "Long spin-relaxation time in a single metal nanoparticle," Nature Nanotechnology, vol. 5, no. 8, pp. 593-596, 2010.

[37] H. Kobayashi, Y. Iida, and Y. Omura, "Single-mode silicon optical switch with T-shaped $\mathrm{SiO}_{2}$ optical waveguide as a control gate," Japanese Journal of Applied Physics, vol. 41, 4B, Part 1, pp. 2563-2565, 2002.

[38] D. K. Schroder, R. N. Thomas, and J. C. Swartz, "Free carrier absorption in silicon," IEEE Journal of Solid-state Circuits, vol. SC-13, pp. 180-187, 1978.

[39] R. A. Soref and B. R. Bennet, "Electrooptical effects in silicon," IEEE Journal of Quantum Electronics, vol. 23, no. 1, pp. 123129, 1987.

[40] R. Terawaki, Y. Takahashi, M. Chihara, Y. Inui, and S. Noda, "Ultrahigh-Q photonic crystal nanocavities in wide optical telecommunication bands," Optics Express, vol. 20, no. 20, pp. 22743-22752, 2012.

[41] R. A. Weeks, "The many varieties of $\mathrm{E}^{\prime}$ centers: a review," Journal of Non-Crystalline Solids, vol. 179, pp. 1-9, 1994.

[42] D. L. Griscom, "Defect structure of glasses: some outstanding questions in regard to vitreous silica," Journal of NonCrystalline Solids, vol. 73, no. 1-3, pp. 51-77, 1985.

[43] H. Imai, K. Arai, H. Hosono, Y. Abe, T. Arai, and H. Imagawa, "Dependence of defects induced by excimer laser on intrinsic structural defects in synthetic silica glasses," Physical Review $B$, vol. 44, no. 10, pp. 4812-4818, 1991.

[44] K. Awazu, H. Kawazoe, K. Harada, K. Kido, and S. Inoue, "Precursor to paramagnetic centers induced in gammairradiated doped silica glasses," Journal of Applied Physics, vol. 73, no. 4, pp. 1644-1649, 1993.

[45] Y. Nishi, "Study of silicon-silicon dioxide structure by electron spin resonance I," Japanese Journal of Applied Physics, vol. 10, no. 1, pp. 52-62, 1971.

[46] E. H. Poindexter, G. J. Gerardi, M.-E. Rueckel, P. J. Caplan, N. M. Johnson, and D. K. Biegelsen, "Electronic traps and $\mathrm{P}_{\mathrm{b}}$ centers at the $\mathrm{Si} / \mathrm{SiO}_{2}$ interface: band-gap energy distribution," Journal of Applied Physics, vol. 56, no. 10, pp. 2844-2849, 1984.

[47] E. Holzenkämpfer, F. W. Richter, J. Stuke, and U. Voget-Grote, "Electron spin resonance and hopping conductivity of aSiOx," Journal of Non-Crystalline Solids, vol. 32, no. 1-3, pp. 327-338, 1979.

[48] Y. Shiraishi, K. Takano, J. Matsubara et al., "Growth of silicon crystal with a diameter of $400 \mathrm{~mm}$ and weight of $400 \mathrm{~kg}$," Journal of Crystal Growth, vol. 229, no. 1-4, pp. 17-21, 2001.

[49] J. Ryuta, E. Morita, T. Tanaka, and Y. Shimanuki, "Crystaloriginated singularities on Si wafer surface after SC1 cleaning," Japanese Journal of Applied Physics, vol. 29, no. 11, Part 2, pp. L1947-L1949, 1990. 
[50] A. Ikari, K. Nakai, Y. Tachikawa et al., "Defect control in nitrogen doped Czochralski silicon crystals," Solid State Phenomena, vol. 69-70, pp. 161-166, 1999.

[51] K. Wada, H. Nakanishi, H. Takaoka, and N. Inoue, "Nucleation temperature of large oxide precipitates in as-grown Czochralski silicon crystal," Journal of Crystal Growth, vol. 57, no. 3, pp. 535-540, 1982. 


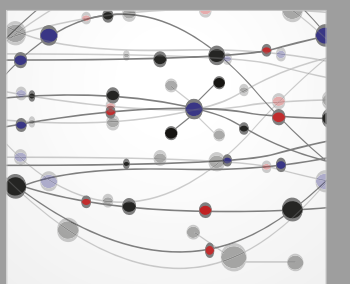

The Scientific World Journal
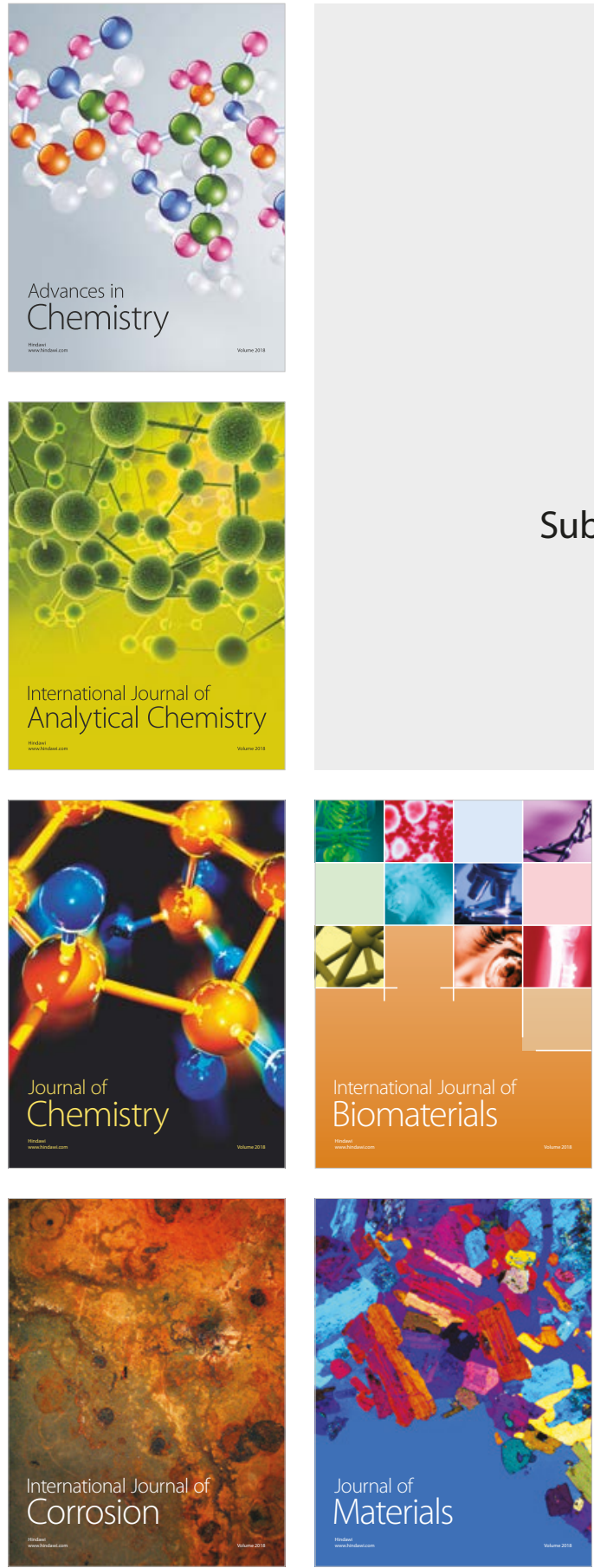

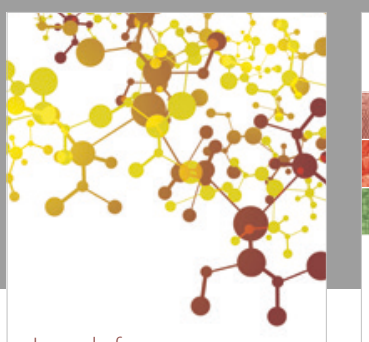

Journal of

Applied Chemistry
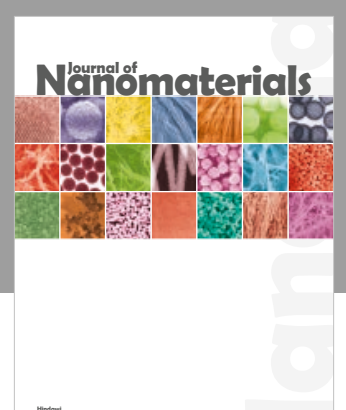

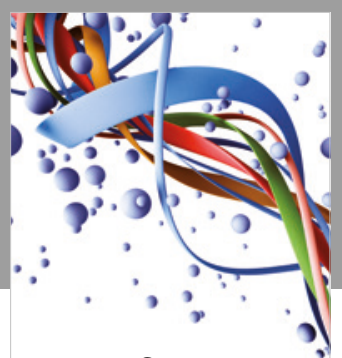

Scientifica

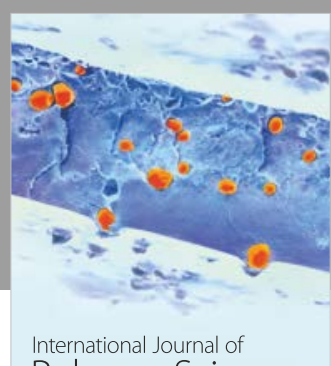

Polymer Science

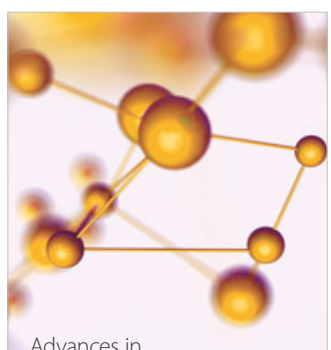

Physical Chemistry
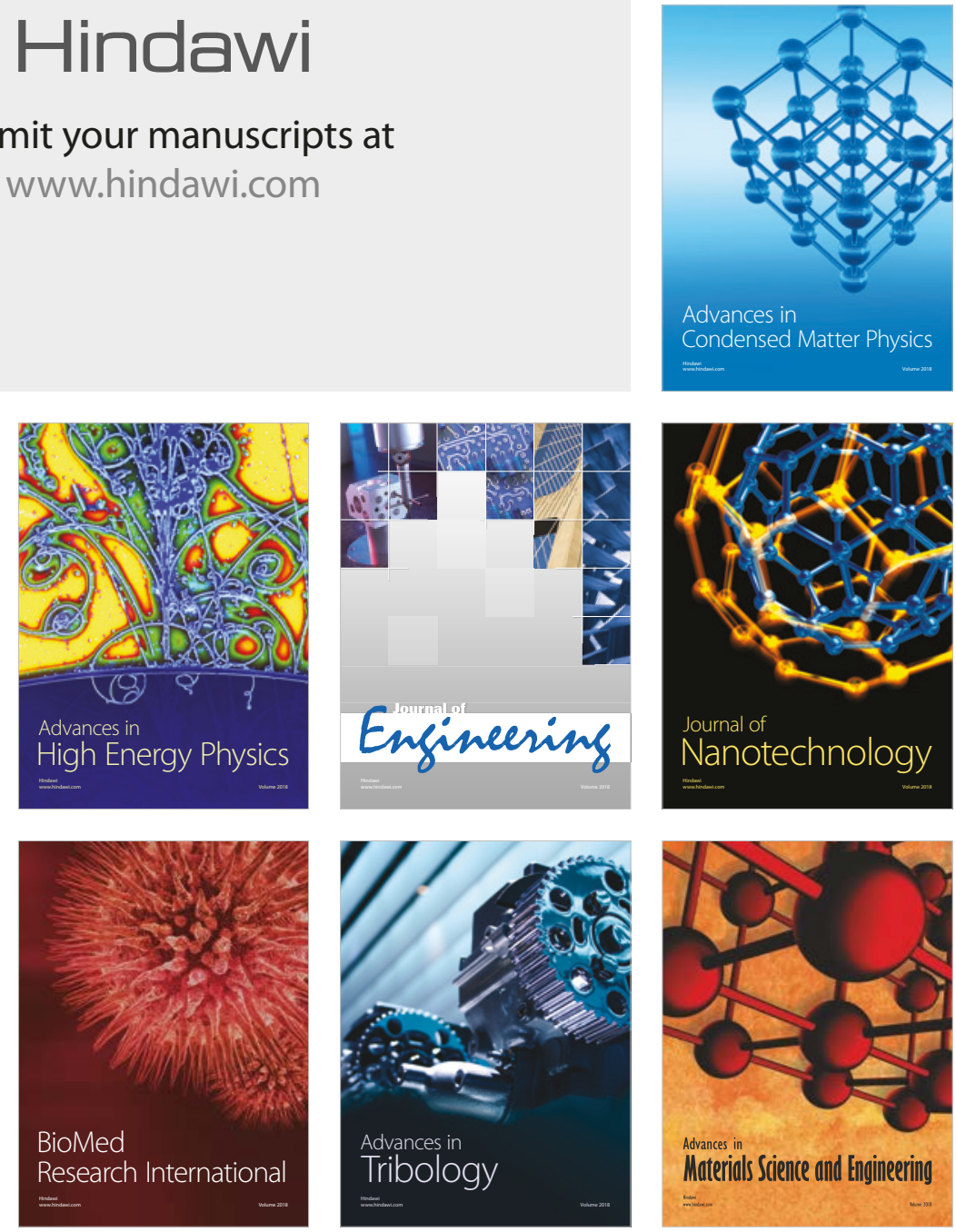